\title{
EL REGISTRO MICROPALEONTOLÓGICO DEL SOLUTRENSE EN LA REGIÓN CANTÁBRICA (ESPAÑA): ESTADO DE LA CUESTIÓN
}

\author{
The micropaleontological record of the Solutrean in the \\ Cantabrian Region (Spain): state of arts
}

\author{
Naroa García-Ibaibarriaga ${ }^{1,3}$, María José Iriarte-Chiapusso ${ }^{2,3}$, Xabier Murelaga ${ }^{1}$
}

Recibido el 15 de marzo de 2013. Aceptado el 11 de julio de 2013

Resumen. El presente artículo es una revisión de los sitios arqueológicos y paleontológicos con estudios palinológicos y de microvertebrados de cronología Solutrense de la región cantábrica. Aunque hoy en día, los estudios paleoambientales son un elemento habitual en las actuaciones arqueológicas, la información correspondiente a este periodo sigue siendo escasa. Además, es importante resaltar que la mayoría de los yacimientos fueron excavados en el siglo pasado, cuando todavía no se aplicaban las actuales técnicas de muestreo. La información palinológica y micropaleontológica nos permite construir el paleoambiente y el clima que existieron en el periodo cronocultural conocido como Solutrense.

Palabras clave: Solutrense, región cantábrica, paleoambiente, Paleolítico superior, palinología, microvertebrados.

Abstract. The main aim of this paper is to present a revision of several Solutrean archaeological and palaeontological sites in the Cantabrian Region with palynological and small vertebrate studies. Even though nowadays the analyses seeking out palaeoenvironmental information are becoming more popular, the information belonging to this period remains still scarce. Although we cannot obviate the fact that most of the archaeological sites were excavated during last century, when they did not apply the same sampling techniques as nowadays, palynological and micropalaeontological data allow us to reconstruct the palaeoenvironment and the climate of the Solutrean period.

Keywords: Solutrean, cantabrian region, palaeoenvironment, Upper Paleolithic, palynology, small vertebrate.

(') Departamento de Estratigrafia y Paleontología. Facultad de Ciencia y Tecnología. Universidad del Pais Vasco UPV/EHU. Barrio Sarriena s/n. E-48940 Leioa.naroa.garcia1@ehu.es·xabier.murelaga@ehu.es.

(2) IKERBASQUE, Basque Foundation for Science. E-48011, Bilbao.

(3) Departamento de Geografía, Prehistoria y Arqueología. Facultad de Letras. Universidad del País Vasco UPV/EHU. Calle Tomás y Valiente s/n. E-01006, Vitoria-Gasteiz.mariajose.iriarte@ehu.es. 


\section{INTRODUCCIÓN: LA REGIÓN CANTÁBRICA}

El interés de los prehistoriadores por conocer la evolución del entorno medioambiental en el que vivieron los moradores de los yacimientos arqueológicos durante el Paleolítico viene de lejos, aunque en un principio solo se centraron en los análisis de los restos faunísticos de mayor tamaño.

Con el paso del tiempo, y gracias al desarrollo y aplicación de una interesante diversidad de analíticas, ha quedado demostrada la importante información que el estudio de los restos microfaunísticos y paleobotánicos aportan sobre la dinámica paleoambiental y paleoclimática. Estas analíticas, al igual que otras, han sufrido una considerable mejora cualitativa, y lo que es más importante, también un avance en su ámbito metodológico.

La región cantábrica comprende todo el territorio situado al norte de la Cordillera Cantábrica, desde Galicia hasta el País Vasco. Administrativamente, forman parte de ella la provincia de Lugo (Galicia), el norte de las provincias de León, Palencia y Burgos (Castilla y León), las comunidades autónomas de Asturias y Cantabria y las provincias de Bizkaia y Gipuzkoa (País Vasco). Debido a la marcada influencia del océano Atlántico (mar Cantábrico), esta zona geográfica presenta un clima de tipo oceánico, caracterizado por la suavidad de sus temperaturas medias, una reducida amplitud térmica y una elevada pluviosidad. Estas condiciones climáticas, junto a la orografía y al tipo de sustrato, constituyen la base de la interesante diversidad biogeográfica de este territorio.

Aun cuando la actividad humana ha modificado considerablemente el paisaje natural, todavía es posible encontrar ejemplos de la diversidad paisajística. En las áreas limítrofes con el mar, las dunas, acantilados, rías y marismas acogen a aquellas especies vegetales adaptadas a elevados índices de salinidad (Zostera, Salicornia, Spartina, etc.). Hacia el interior del piso colino y hasta unos 500-600 m de altitud, los prados de origen antrópico ocupan gran parte de la región. El bosque mixto de caducifolios (roble, avellano, fresno, abedul, tilo, castaño, etc.) y su rico cortejo arbustivo, son los que más han sufrido la acción humana y compiten duramente con los bosques plantados con fines económicos, centrados sobre todo en dos especies, el eucalipto y cada vez menos, en el pino. En aquellas zonas de afloramiento calizo donde la permeabilidad del agua supone un mayor grado de aridez del sustrato se desarrollan los encinares cantábricos, en los que a la encina le acompañan especies de ámbito mediterráneo como el madroño o aladierno, junto a otras de carácter marcadamente atlántico como el avellano o el espino albar. Dentro de los bosques de ribera, destacan los alisos y los sauces, quienes junto a otras especies como el fresno u olmo generan un amplio cortejo herbáceo-arbustivo que enriquecen las orillas de los ríos cantábricos. En el piso montano, la orografía reduce la amplitud de los prados anteriormente mencionados, dándose un mayor predominio del matorral. Las zonas más boscosas suelen ser aquellas de más difícil acceso, donde robledales, hayedos, abedulares, quejigales, etc. comparten el área en función de sus características ecológicas. En aquellas montañas de la Cordillera Cantábrica de altitud superior a los 1600 m, nos encontraremos también una vegetación subalpina caracterizada por los pastizales y matorrales de alta montaña (por ejemplo, enebro rastrero, gayuba o torvisco), que en altitudes superiores a $2200 \mathrm{~m}$ dan lugar a pastizales alpinos naturales y vegetación de roquedo.

Entre las especies de micromamíferos presentes hoy en día en la región cantábrica, Microtus (Microtus) arvalis, Microtus (Microtus) agrestis, Crocidura suaveolens, Sorex coronatus y Talpa europaea predominan en contextos de pradera. En entornos boscosos, normalmente encontramos a especies como Apodemus flavicollis, Glis glis, Sciurus vulgaris y/o Clethrionomys glareolus y zonas próximas al agua Neomys fodiens y Galemys pyrenaicus.

En este texto, se presenta el estado de la cuestión actual respecto a la información paleoambiental obtenida a

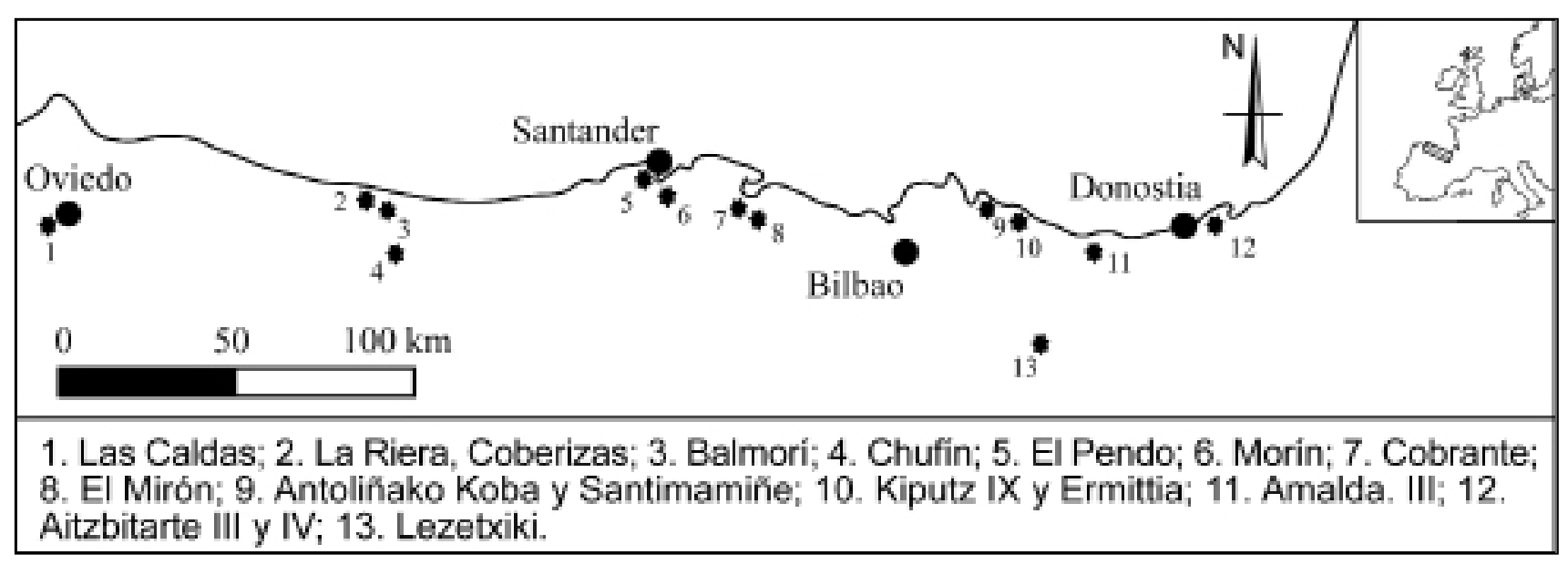

$\Delta$ Figura 1. Localización geográfica de los yacimientos mencionados en el texto. 
partir del registro micropaleontológico durante el periodo cronológico coincidente con el desarrollo de la cultura solutrense. Dentro del marco geográfico seleccionado, existen diversos depósitos en cuyas secuencias cronoestratigráficas, hay niveles solutrenses. La mayoría de ellos, corresponden a yacimientos arqueológicos, disponiendo de una mermada representación de los depósitos de origen no antrópico. Del total de secuencias del periodo que nos interesa, únicamente, en 17 de ellas se han realizado estudios micropaleontológicos (paleopalinológicos y/o microfaunísticos): cuevas de Las Caldas, La Riera, Coberizas y Balmori en Asturias; cuevas de Chufín, El Pendo, Morín, Cobrantes y El Mirón en Cantabria y las cuevas de Santimamiñe, Lezetxiki, Antoliñako Koba, Kiputz IX, Ermittia, Amalda, Aitzbitarte IV y Aitzbitarte III en el País Vasco (Fig. 1).

\section{REGISTROS MICROPALEONTOLÓGICOS DISPONIBLES}

En este apartado exponemos un sucinto comentario de cada uno de los depósitos incluidos en el texto. Además, se incluyen las principales citas bibliográficas para cada yacimiento a fin de evitar ulteriores reiteraciones.

\subsection{Gipuzkoa}

\section{Aitzbitarte III (Rentería)}

La cueva de Aitzbitarte III se localiza sobre el torrente de Landarbaso (cuenca del Urumea), a 220 m sobre el nivel del mar (s.n.m). En las excavaciones realizadas en la entrada de la cueva se ha descubierto una interesante secuencia del Paleolítico superior, en la que destacan las ocupaciones auriñacienses y gravetienses. Los niveles III y II de este yacimiento, parece que se sitúan en un momento a caballo entre el final del Gravetiense y el Solutrense medio (Altuna et al. 2011). El estudio palinológico de estos niveles ha presentado problemas de conservación esporopolínica (Iriarte-Chiapusso 2011a). En el material de microfauna, está compuesto por un total de 287 restos pertenecientes a las especies Talpa sp., Sorex gr. araneus, Arvicola terrestris, Microtus gr. agrestis-arvalis, Microtus nivalis y Microtus oeconomus (Peman 2011).

\section{Aitzbitarte IV (Rentería)}

La cueva de Aitzbitarte IV se ubica algunos metros por encima de la cueva de Aitzbitarte III. Fue excavada entre 1960-1964 por J.M. de Barandiarán (1961, 1963a y b 1964, 1965). J. Altuna (1970), identifica las siguientes especies en el nivel Solutrense medio y superior (IV): Talpa europaea, Sorex araneus, Arvicola terrestris, Pitymys sp., Microtus ratticeps, Microtus gr. agrestis-arvalis y Microtus nivalis.

\section{Amalda (Zestoa)}

Esta cueva de la cuenca del río Urola (valle del río Alzolaras), se sitúa a $205 \mathrm{~m}$ s.n.m En su secuencia (Altuna et al. 1990) hay constancia tanto de ocupaciones paleolíticas (desde el Paleolítico medio al Solutrense) como holocenas (Calcolítico y Tardorromano). El estudio palinológico fue realizado por M. Dupré (1988, 1990). En el estudio de microfauna del nivel IV (Solutrense superior) realizado por E. Peman (1990), se identifican 5 especies pertenecientes al Orden Soricomorpha, 10 al Orden Rodentia y uno al Orden Chiroptera.

\section{Kiputz IX (Mutriku)}

El depósito de Kiputz IX se sitúa al este del monte Arno (119 m s.n.m). La principal característica de este depósito es que esta cavidad ha funcionado como una trampa natural para los animales durante el Pleistoceno superior, desde el Estadio Isotópico 3 (Castaños 2005, 2006, 2007 y 2008). En la actualidad, se están realizando las diferentes analíticas cuyos resultados preliminares se incluyen en este texto (M.J. IriarteChiapusso) y remitimos al lector al artículo de esta misma monografía (Garcia-lbaibarriaga et al. en este volumen) para las referencias sobre los restos de micromamíferos.

\section{Ermittia (Deba)}

Este yacimiento, descubierto en 1924 por J.M. de Barandiarán, quien lo excavó junto con T. de Aranzadi, entre 1924-1926, es una de las tres cuevas que se encuentran en la ladera oeste del monte Ermittia (100 m s.n.m.). La ocupación solutrense (nivel V) tiene una potencia de unos $30 \mathrm{~cm}$ y en él, J. Altuna (1972) señala la presencia de Talpa europaea, Lepus sp., Arvicola terrestris, Microtus oeconomusy Microtus gr. agrestis-arvalis.

\section{Lezetxiki (Arrasate)}

La cavidad de Lezetxiki se ubica en el valle del río Deba, en la cuenca fluvial del río Kobate, a 375 m s.n.m. La cavidad presenta una morfología característica, tipo túnel con una orientación aproximada norte-sur que se mantiene en el buzamiento sedimentario. Presenta una amplia secuencia estratigráfica (más de 9 metros) que se inicia en el Paleolítico inferior y culmina en el Holoceno (Barandiarán y Altuna 1970; Arrizabalaga et al. 2005). En los niveles que nos interesan, tanto en el estudio palinológico realizado por M.F. Sanchez-Goñi (1993), como en el de M.J. Iriarte-Chiapusso (inédito), hay problemas de conservación esporopolínica. La información disponible sobre los micromamíferos procede del estudio que realizó J. Altuna (1972) sobre el material faunístico procedente de las excavaciones de J.M. de Barandiarán. Carecemos de información sobre los micromamíferos, ya que en el listado faunístico del nivel II no se mencionan este tipo de restos. 


\subsection{Bizkaia}

\section{Antoliñako Koba (Gautegiz-Arteaga)}

Esta cavidad, localizada en la vertiente sur del monte Arlanburu (Parque Natural del Urdaibai), a 300 m s.n.m, fue excavada por J.M de Barandiarán en 1917, 1923 y 1936 (Barandiarán 1925 y 1947). Entre 1995-2000 se realizó una nueva actuación arqueológica bajo la dirección de $M$. Aguirre (2000) en la que ha diferenciado 4 unidades estratigráficas, cronológicamente situadas entre 12080-11768 y 35096-34090 cal BP ' . El nivel C tiene una industria asociada al Solutrense superior, y a su vez, está dividido en dos subniveles. La zona media del subnivel C2 está datada en 23435-22515 cal BP (19280 \pm 120 BP). La deficiente conservación esporopolínica de los niveles estudiados hasta el momento (Iriarte-Chiapusso, inédito), imposibilita disponer de información sobre el paisaje vegetal durante este periodo. H. Zubeldia (Zubeldia 2006 y Zubeldia et al. 20032007) ha estudiado dos muestras del nivel $C_{\text {, en las que }}$ se describe las siguientes especies: Terricola sp., Apodemus sylvaticus-flavicollis, Sorex sp., Crocidura sp., Talpa sp. y Chionomys nivalis.

\section{Santimamiñe (Kortezubi)}

La cueva de Santimamiñe se localiza en la margen oriental de la cuenca de Urdaibai, en la ladera sur del monte Ereñozar (150 m s.n.m). El santuario rupestre fue descubierto en 1916 y la excavación arqueológica se desarrolló en dos fases: 1918-1926 y 1960-1962 (Barandiarán 1976). Entre 2004-2006, bajo la dirección de J.C. López Quintana (2011), se ha realizado una revisión estratigráfica y nuevas analíticas. La secuencia se inicia en un nivel paleontológico (Arb-o / 31481-31055 cal BP: $26890 \pm 180$ BP) y finaliza en un nivel histórico (época romana). En el nivel paleontológico (Arg-o), posiblemente originado por la acción de algún animal depredador, hay restos de caballo, gran bóvido, ciervo, reno, sarrio y oso pardo. Sobre este nivel, existe un importante nivel sedimentario denominado Complejo hidrológico que se enmarca entre el nivel Arg-o (24956-24170 cal BP -20530 \pm 110 BP-) y el nivel Camr (18424-17486 cal BP $-14650 \pm 80$ BP-), que ha resultado esporopolínicamente estéril (Iriarte-Chiapusso 2011b). Los restos de micromamiferos aparecen en el nivel Arg-o y en la zona superior del Complejo hidrológico (Lsr-Ap), situado debajo del nivel Camr. Las especies identificadas son: Microtus (Terricola) sp., Chionomys nivalis, Microtus (Alexandromys) oeconomus, Microtus (Microtus) agrestis-arvalis, Apodemus sp., Sorex coronatus-araneus y Talpa sp. (Murelaga et al. 2011).

\subsection{Cantabria}

\section{Cobrante (San Miguel de Aras)}

La cueva de Cobrante está situada en la ladera del Mazo de Cobrante en el valle de Aras, a 150 m s.n.m. Excavada desde 1998 bajo la dirección de Pedro Rasines, su registro sedimentario contiene diversos niveles adscritos al Aziliense, Solutrense y Auriñaciense. En este depósito disponemos de sendos estudios de palinología y microfauna. El primero ha sido realizado por B. Ruiz Zapata y M.J. Gil Garcia (2009) y de los niveles solutrenses sólo se dispone de una muestra (nivel 3), ya que el nivel 4 ha resultado estéril. C. Sesé (2009) en el estudio micropaleontológico ha identificado las siguientes especies: Apodemus sp., Arvicola terrestris, Microtus arvalisagrestis, Microtus oeconomus, Microtus Iusitanicus, Sorex araneus, Neomys fodiens, Talpa europaea y Lepus sp.

\section{El Mirón (Ramales de la Victoria)}

La gran cueva de El Mirón se localiza en la cara oeste del Monte Pando, en un valle encajonado donde los ríos Gándara y Calera se unen (altitud: $250 \mathrm{~m}$ s.n.m). Dispone de una amplia secuencia estratigráfica, que se inicia en la transición del Paleolítico medio al superior y culmina en época histórica. Una vez finalizado el estudio paleopalinológico de los niveles holocenos (Iriarte-Chiapusso 2012), en la actualidad se está ultimando la secuencia pleistocénica, en la que se incluyen los niveles solutrenses (Straus et al. 2011; Straus et al. en prensa). El estudio de microfauna de los niveles solutrenses se ha realizado en dos zonas diferentes de la cueva (zona de la Trinchera y zona de la Rampa). En él se han identificado las siguientes especies (Cuenca-Bescos et al. 2008 y 2009): Pliomys lenki, Arvicola terrestris, Terricola lusitanicus, Microtus agrestis, Sorex gr. coronatus-araneus, Neomys fodiens, Talpa europaea, Mustela nivalis, Microtus oeconomus, Sorex minutus, Microtus arvalis, Chionomys nivalis y Chiroptera indet.

\section{Morín (Villaescusa)}

La cueva de Morín, también conocida como Cueva del Rey, se encuentra en un amplio valle, flanqueado al este por la sierra de Peña Cabarga, a 50 m s.n.m. Su completa secuencia cultural (González Echegaray y Freeman 1971) se inicia en el Paleolítico medio (Musteriense - nivel 22) y culmina en un nivel con ocupación aziliense (nivel 1). El estudio palinológico fue realizado por Arl. Leroi-Gourhan (1971), aunque, recientemente, en el marco de una nueva actuación desarrollada con el objetivo de incidir en la investigación geomorfológica y paleoambiental de este yacimiento, dirigida por J. M. Maíllo (Maillo et al. 2001) se ha realizado un nuevo mues-

(1) La calibración de las dataciones se ha efectuado con el programa/software OxCal 4.1.7. (Bronk 2009), basado en la curva de Calibración INTCAL09 (Reimer et al. 2009). 
treo palinológico (Iriarte-Chiapusso inédito). Únicamente se menciona la presencia de Talpa sp. y Arvicola sp. (3 y 2 restos respectivamente que corresponderian a un individuo mínimo cada uno) en contextos solutrenses (Altuna 1971).

\section{El Pendo (Escobedo de Camargo)}

La secuencia cultural de la cueva de El Pendo, cavidad sitauada a 25 m s.n.m, se inicia durante el Paleolítico medio (González Echegaray 1980). El estudio palinológico fue realizado por Arl. Leroi-Gourhan (1980). En cuanto a los micromamíferos, el estudio paleontológico no se menciona la presencia este tipo de restos (Altuna 1972).

\section{Chufín (Riclones)}

La cueva de Chufín, también conocida como Moro Chufín, se sitúa en el barranco del río Lamasón, en las proximidades de su confluencia con el río Nansa, a una altitud de 150 m s.n.m. Este es el único yacimiento arqueológico de esta exposición que solamente se ocupó en un periodo cultural, el Solutrense. Este nivel arqueológico está enmarcado por sendos niveles sedimentarios que carecen de registro antrópico (Cabrera 1977). El estudio palinológico fue realizado por A. Boyer-Klein (1980 y 1984). No hay información sobre restos de micromamíferos.

\subsection{Asturias}

\section{Balmori (Balmori)}

Este yacimiento cuya secuencia se inicia en el Solutrense, se encuentra a $40 \mathrm{~m}$ s.n.m, en las cercanías del estuario de Barro. J. Menéndez Amor analizó unas muestras que contenían una baja densidad de polen (Clark 1974). Dentro del estudio paleontológico realizado por J. Altuna (1972) sobre el material procedente de las catas efectuadas por A. Clark en 1969, identificó Talpa europaea, Arvicola terrestris y Apodemus sp.

\section{Coberizas (Llanes)}

La cueva de Coberizas, fue descubierta por H. Obermaier y el conde de la Vega del Sella en 1920. G.A. Clark realizó algunas catas en 1969, diferenciando diversas ocupaciones (Solutrense, Magdaleniense tardío o Aziliense y Asturiense). J. Altuna (1972) estudió el material procedente de las catas de G.A. Clark e identificó restos de Talpa europaea, Arvicola terrestris, Microtus ratticeps, Microtus gr. agrestis-arvalis.

\section{La Riera (Posada de Llanes)}

El yacimiento de La Riera se sitúa a unos 40 m s.n.m, el cual se encuentra en la actualidad a 1,5 km de distancia. Esta cavidad ha sido ocupada en diferentes periodos del Paleolítico superior y Aziliense (Straus y Clark 1986), siendo los niveles 2/3 al 17 los que comprenden la ocupación solutrense. El estudio palinológico fue realizado por Arl. Leroi Gourhan
(1986). En un primer momento, J. Altuna (1986), menciona las siguientes especies: Erinaceus europaeus, Talpa europaea, Talpa caeca, Galemys pyrenaicus, Lepus sp., Oryctolagus cuniculus, Arvicola terrestris, Arvicola sapidus, Microtus oeconomus, Microtus agrestis-arvalis, Pitymys sp. Posteriormente, J.M. López-García en su Tesis Doctoral (2008) solo menciona la presencia de Talpa europaea, Galemys pyrenaicus, Erinaceus europaeus, Microtus oeconomus y Terricola sp.

\section{Las Caldas (San Juan de Priorio)}

La cueva de Las Caldas, ubicada en un valle lateral del margen izquierdo del río Nalón (160 m s.n.m), tiene una amplia secuencia estratigráfica con niveles solutrenses y magdalenienses (Corchón 1981 y 1995). El estudio palinológico de los tramos II y IV de la unidad I fue realizado por M. Paquereau (1981). En la publicación preliminar del análisis de micromamíferos (Laplana et al. 2006), se indica la presencia de Arvicola terrestris, Arvicola sapidus, Microtus arvalis, Microtus agrestis, Microtus oeconomus, Microtus lusitanicus, Chionomys nivalis, Pliomys lenki (cita más moderna), Eliomys quercinus, Sorex gr. araneus, Sorex minutus, Talpa europaea, Neomys fodiens, Lepus timidus (cita más occidental) y Myotis myotis. Las especies dominantes, tanto en el talud exterior (Solutrense superior), como en la Sala I (Solutrense inferior, medio y superior) serían Arvicola terrestris y Microtus arvalis, especies típicas de zonas de temperaturas estivales relativamente bajas.

\section{ESTADO DE LA CUESTIÓN}

Una vez revisada la información paleoambiental disponible, el principal problema que nos encontramos a la hora de establecer la seriación medioambiental del Solutrense, es la escasez de registros continuos. En esta circunstancia confluyen diversos factores.

En primer lugar, uno de los principales condicionantes existentes son los procesos erosivos postdeposicionales que afectan a la secuencia estratigráfica del depósito, como sucede, por ejemplo, en el caso de la Cueva de El Otero, donde al nivel 5 adscrito al Auriñaciense, le sucede un nivel Magdaleniense -nivel 6- (González Echegaray et al. 1966). 0 en la cueva de Amalda, donde al nivel solutrense (nivel IV) le sucede un nivel Calcolítico (III). Además, a veces, el objetivo de la analítica se centra en una zona concreta de la secuencia (Balmori, Las Caldas).

Otro factor que limita la seriación continua de los registros solutrenses es la deficiente conservación de los restos. En ocasiones, éste problema puede afectar a unos niveles concretos, a determinados lechos o a todo el registro del depósito (por ejemplo, Lezetxiki, El Mirón, Santimamiñe o Kiputz IX).

En el caso concreto de los estudios paleobotánicos, también hay que tener en cuenta aquellas muestras que, aunque conserven restos paleobotánicos, no resultan estadística- 
mente válidos. En la actualidad, se consideran estadísticamente válidas aquellas muestras que contienen un mínimo de 250 pólenes (Burjachs et al. 2003), sin contar las esporas de helechos, y al menos 15 taxones diferentes. En algunos de los estudios publicados, existen muestras que no alcanzan o superan escasamente los 100 pólenes, por lo que no han sido incluidas en esta revisión (por ejemplo, Chufín y La Riera).

Un factor destacado que resulta ser un importante condicionante de los resultados obtenidos es la estrategia de muestreo. El objetivo de todo estudio de una secuencia estratigráfica es el de obtener unos datos que resulten fiables cualitativa y cuantitativamente. La interrelación de las diversas analíticas aplicadas debe realizarse desde el mismo momento en que se elabora el proyecto de investigación, estableciéndose una estrategia de muestreo común, sin olvidar los requerimientos específicos de cada analítica. A ser posible conviene que los diferentes muestreos se realicen en una misma zona, o por lo menos intentar que abarquen la misma seriación estratigráfica.

En todo muestreo, la distancia con que se recogen las muestras (intervalo de muestreo) tiene que ser lo más ajustada posible, dado que un amplio intervalo puede enmascarar cambios medioambientales. En el estudio palinológico de la cueva de El Pendo, se siguió el criterio geológico de recoger una muestra por nivel sedimentario, lo que condicionó los resultados. Con posterioridad se ha repetido el análisis, aunque el sedimento ha resultado ser esporopolínicamente estéril.

La metodología utilizada a la hora de recuperar los restos de microvertebrados ha resultado ser un aspecto muy condicionante de los resultados obtenidos, ya que dependiendo del tipo de luz de malla utilizado en la criba del sedimento (tanto en seco como con agua), obtendremos unos resultados $u$ otros. En la mayoría de las excavaciones realizadas en los años 80 y 90 el sedimento era cribado usando mallas de $2-3 \mathrm{~mm}$ de luz y únicamente se recogian los fósiles de visu. De esta forma, se perdían los elementos de menor tamaño y se sobre representaban los de mayor talla. Como ejemplo de ello, destacar una prueba que se hizo con los

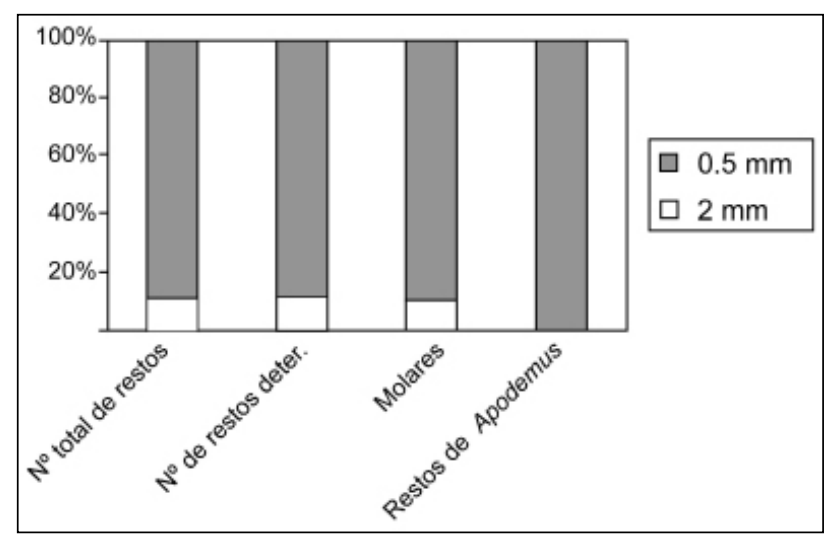

A Figura 2. Santimamiñe: comparación de muestreo utilizando tamiz de $2 \mathrm{~mm}$ y de $0.5 \mathrm{~mm}$. materiales del yacimiento de Santimamiñe. La grafica de la figura 2 corresponde al total de muestras recogidas en el cribado, pero se han contabilizado independientemente las recogidas en el tamiz de $2 \mathrm{~mm}$ y las del tamiz de $0.5 \mathrm{~mm}$ de luz de malla. Se puede observar la cantidad de restos que se pierden si solo utilizamos el tamiz de $2 \mathrm{~mm}$, y es de destacar que un taxón importante en la determinación paleoambiental en la región cantábrica, como es el género Apodemus, no estaría representado en la muestra a estudiar.

Por último, la información que disponemos se encuentra limitada por la publicación de un modo parcial y/o incompleto de las analíticas. De todos modos, a pesar de todas estas limitaciones, la dinámica actual de las investigaciones nos está permitiendo incrementar nuestra información sobre el entorno paleoambiental del estadio isotópico que nos ocupa (OIS2).

\section{CONTEXTUALIZACIÓN PALEOCLIMÁTICA}

\subsection{El paisaje vegetal}

Nuestro conocimiento de la evolución del paisaje vegetal durante el Solutrense en la región cantábrica (teniendo en cuenta los factores mencionados en el apartado 3) se restringe a los datos procedentes de las cavidades de La Riera, Cobrante, El Mirón, Kiputz IX, Amalda y Chufín. De estos depósitos, las secuencias solutrenses más amplias corresponden a los yacimientos arqueológicos de La Riera y El Mirón. Sin embargo, la escasez de restos recuperados en algunas de sus muestras, interrumpen la visión continua de ambos registros. En el caso de la cueva de La Riera, la totalidad de las muestras han sido representadas en el estudio y diagrama polínicos, aunque en 12 de ellas no se alcanzan los 150 palinormofos. Si nos atenemos al criterio actual de considerar una muestra estadisticamente válida cuando alcanza al menos los 250 pólenes (sin contar las esporas), el registro de La Riera quedaría reducido a 5 muestras. En esta exposición, y dada la reducida disponibilidad de datos, vamos a tener en consideración todas las muestras de este depósito que superen los 150 restos polínicos.

El primer nivel solutrense de La Riera, cuyo registro palinológico cumple con el criterio expuesto, es el nivel 7. En este momento posterior al 26921-23578 cal BP (nivel 4: $20970 \pm 620$ BP), existe un paisaje abierto en el que el brezal tiene una importante presencia (en torno al $50 \%$ ). La reducida cubierta arbórea (inferior al $7 \%$ ) tiene como principales componentes al pino, enebro y avellano, aunque entre la vegetación de ribera también aparecen aliso y sauce. No obstante, estos taxones caducifolios desaparecen en la muestra más reciente del siguiente nivel (8), lo que unido a la dinámica del resto de los componentes de este registro, reflejan un deterioro de las condiciones climáticas que limita aún más el territorio del bosque (Pinus y Juniperus). 
A partir del nivel 9 se detectan unos periodos de mejoría climática (niveles 9, 13, 14 -muestra superior- y 16: 2337020290 cal BP, $18200 \pm 610$ BP) en los que progresivamente el estrato arbóreo aumenta su extensión. Aunque sus valores porcentuales no llegan a superar el $10 \%$, en estos momentos los taxones caducifolios (avellano, abedul, aliso y sauce) se recuperan y Quercus ilex aparece en la secuencia. Los brezos siguen dominando en el estrato herbáceo-arbustivo (superan en más de una ocasión el $50 \%$ ). No obstante, entre estos periodos existen pulsaciones de regresión de las condiciones climáticas, como sucede en el nivel 10 (24672-22530 cal BP: $19820 \pm 390$ BP) o en la base del nivel 14 .

La muestra correspondiente al nivel paleontológico de Santimamiñe (24956-24170 cal BP) tiene uno de los mayores valores porcentuales de polen arbóreo (23\%), aunque Pinus es su principal componente (Juniperus no alcanza el $3 \%$ ). La composición del estrato herbáceo-arbustivo, junto a la dinámica de los helechos y la ausencia de elementos estépicos, indicarian que en este periodo las condiciones paleoclimáticas eran frías pero relativamente húmedas.

El nivel F del depósito paleontológico de Kiputz IX tiene dos dataciones de C14 que sitúan su zona media entre 24237-23330 cal BP $(19870 \pm 150$ BP $)$ y la zona superior entre 22084-21369 cal BP (18140 \pm 90 BP). Los resultados preliminares del estudio palinológico evidencian una deficiente conservación esporopolínica en la base de la secuencia. El pino, al igual que en el sitio precedente, es el único referente arbóreo constante de la secuencia, mientras que el abedul y/o enebro le acompañan de un modo discontinuo. En el entorno medioambiental de Kiputz IX existía un paisaje abierto con escasa cubierta forestal, en el que la dinámica de los taxones herbáceos y de las esporas de helechos parecen sugerir que no existía un grado de humedad (los brezos no alcanzan la representación que tienen en La Riera).

La secuencia solutrense del yacimiento de El Mirón, se inicia en torno al 23356-22546 cal BP (nivel 127: $19230 \pm$ $50 \mathrm{BP})$. El paisaje vegetal refleja unas condiciones climáticas frías y secas que se mantienen hasta la muestra más antigua del nivel 126. La cubierta arbórea ronda el $5 \%$ y el pino es su único componente. En el estrato herbáceo-arbustivo predomina Compositae, y aunque las gramíneas incrementan su presencia en el paisaje abierto, la dinámica de los brezos y de los helechos, junto a la presencia esporádica de Artemisia y Centaurea, indica un descenso del grado de humedad. A partir de la zona superior del nivel 126 (23542-21638 cal BP: $18950 \pm 350 \mathrm{BP}$ ) hay un claro cambio en la dinámica paleoclimática. La humedad se recupera (ascenso continuado e importante de brezos, gramíneas, taxones higrófilos y helechos; descenso de Compositae y desaparición de los escasos taxones estépicos) y se recupera la cubierta forestal (AP máximo: $20 \%$ apareciendo el abedul como acompañante del pino. La única muestra del nivel solutrense más reciente (nivel 121: 22653-21213 cal BP, $18390 \pm 300$ BP) parece mantener la misma dinámica, pero el deterioro que sufren los pólenes no nos permite ser más precisos.

El único registro polínico de la cueva de Cobrante (nivel 3: 22431-21602 cal BP, $18540 \pm 70 \mathrm{BP}$ ) refleja una recuperación de las condiciones climáticas que se refleja en la reaparición de Corylus, Quercus caducifolio, Ulmus y Alnus en su cubierta arbórea, cuyo valor porcentual supera el 35 $\%$. En él, pinos y cupresáceas rondan el $25 \%$, el bosque caducifolio supera el $15 \%$ y en menor proporción, los bosques perennifolios y de ribera. En el estrato herbáceo reaparecen los taxones nitrófilos y cosmopolitas y no se observan diferencias significativas en la dinámica de los taxones acuáticos y de las esporas de helechos.

La ocupación solutrense de la cueva de Amalda comienza con un episodio de deterioro climático que supone un retroceso de la cubierta forestal respecto a niveles precedentes, aunque en comparación con los registros anteriormente comentados los valores porcentuales de polen arbóreo son mayores (en torno al $10 \%$ ). El pino es el elemento destacable, mientras que Juniperus, Corylus y Alnus tienen una presencia puntual. La dinámica de los principales componentes del estrato herbáceo-arbustivo (Poaceae y Compositae), la aparición de algunos elementos estépicos como Ephedra y el declive de los helechos, indican el mayor grado de sequedad de este periodo. Estas condiciones varían en la zona superior del nivel IV (22310-20430 cal BP, $17580 \pm 440$ BP, 20210-18679 cal BP, $16200 \pm 380$ BP, y 19831-18741 cal $B P, 16090 \pm 240$ BP), donde los valores de polen arbóreo se llegan a duplicar (Corylus, Alnus, Quercus tp. pedunculado, Betula, Juniperus y Ulmus) y sobre todo se produce la mayor diversidad de taxones de toda la secuencia pleistocena. Es evidente que se produce una mejora de las condiciones climáticas que también afectan al grado de humedad.

El único nivel de ocupación humana de la cueva de Chufín adscrito al Solutrense superior se sitúa cronológicamente entre 21350-20259 cal BP (nivel I: $17420 \pm 200$ BP). En el registro polínico nos volvemos a encontrar el problema de la validez estadística de las muestras. De las cinco muestras que componen este nivel, únicamente la más reciente alcanza los 190 pólenes, mientras que el resto oscila entre 75 y 105 pólenes. A pesar de esta situación, un aspecto relevante en toda la secuencia es la presencia continua de caducifolios (Corylus, Betula, Quercus, Carpinus, Ulmus, y Tilia), aunque hacia el final del nivel parece producirse una ligera regresión. Si nos atenemos a los valores porcentuales más fiables de este registro solutrense (muestra 8), los valores de polen arbóreo son los más elevados de todos los registros comentados (superiores al $30 \%$ ) junto a los valores de esporas de helechos.

No queremos finalizar este apartado, sin hacer mención a un depósito de origen no antrópico localizado al pie de las estribaciones de la Serra do Xistral (Muras, Lugo), denominado Río Bóo, dada la escasez de este tipo de registros en el marco geográfico que nos ocupa para cronologías contem- 
poráneas al Solutrense. Dentro de la secuencia sedimentaria de este depósito, existe una capa limnética de $75 \mathrm{~cm}$ de potencia cuya sedimentación debió producirse en un medio acuático continental debido a la gran cantidad de restos vegetales de taxones acuáticos, como Sphagnum o Cyperaceae que conserva. Se dispone de una datación de C14 ubicada a 20 centímetros de la base: $24131-22671$ cal BP (19680 \pm 220 BP). En este registro polínico (Gómez-Orellana 2002), al igual que en los depósitos anteriores, hay un absoluto predominio del polen no arbóreo. En este caso, las gramíneas destacan ampliamente sobre el resto de los taxones herbáceos. La cubierta forestal evidencia una escasa pero continua representación de Pinus, Betula, Quercus robur tp. o Corylus junto a curvas discontinuas de Fagus y Alnus. El carácter de medio acuático continental queda plenamente reflejado en la variedad de taxones propios de medios semiterrestres y en la dinámica de taxones asociados a medios con mayor presencia de agua como Ranunculaceae, Cyperaceae, Potagometon, Isoetes o Sphagnum. Las condiciones climáticas en las que se desarrolló este paisaje vegetal responderían a unas condiciones frías, que no imposibilitaron el desarrollo de ciertos taxones arbóreos en áreas de mayor refugio, que en opinión del autor del estudio se localizarian en las inmediaciones del depósito.

La primera característica común a todos los depósitos es el importante dominio de los paisajes abiertos, donde gramíneas y brezos mantienen destacadas dinámicas, incluso en los momentos de mayor recrudecimiento climático. En estos periodos, que suelen ir acompañados de un descenso de la humedad, es cuando generalmente Compositae aumenta, llegando a superar a las gramíneas. Pero el descenso de la humedad parece ser que no fue lo significativamente destacado como para permitir la expansión de los taxones estépicos (por ejemplo, Artemisia y/o Ephedra), los cuales, incluso en los momentos de mayor rigurosidad climática, tienen una escasa y discontinua presencia (generalmente, en periodos de mejoría no aparecen en los registros). Las variaciones en el grado de humedad ambiental quedan bien reflejadas en las dinámicas de los helechos y de los taxones higrófilos.

Los valores porcentuales de polen arbóreo rara vez alcanzan el $20 \%$ (la media ronda el $10 \%$ ), a excepción de la muestra más reciente de cueva Chufín y la muestra del nivel 3 de Cobrante. El pino es el taxón arbóreo constante en todos los registros, variando su representación en función de los cambios en las condiciones climáticas. Cuando éstas empeoran el pino puede llegar a ser el único componente de la reducida cubierta forestal, aunque en ocasiones Juniperus puede acompañarle. No obstante, otro patrón coincidente es la aparición de taxones caducifolios en los periodos de cierta recuperación climática (Corylus, Betula, Alnus, Quercus o Ulmus, por ejemplo).

En aquellos registros que disponen de información palinológica anterior al periodo que nos ocupa, (por ejemplo, El
Mirón) se observa que existe una regresión paleoclimática en los primeros niveles solutrenses, que posteriormente tiende a recuperarse. La ausencia de secuencias sedimentarias continuas y $\sin$ hiatos polínicos no nos permite conocer la totalidad de la evolución de la vegetación durante el periodo que nos interesa. Sin embargo, los datos disponibles confirman la existencia de variaciones paleoambientales a lo largo del mismo. Además, no debemos olvidar que factores como la altitud, latitud, orientación, distancia al mar, orografía, etc. aportan diferentes matices a los registros. Posiblemente, la situación geográfica de la cueva de El Mirón influya en el menor grado de humedad que se observa frente al resto de los depósitos más próximos al mar, aunque la línea de costa no estuviera en sus niveles actuales.

\subsection{La microfauna}

La información disponible sobre la fauna de microvertebrados proviene de 14 depósitos. Sin embargo en Aitzbitarte IV, Ermittia, Lezetxiki, Morín, La Riera, Balmori y Coberizas las referencias a este tipo de restos se mencionan dentro del estudio paleontológico. Es decir, se estudiaron los restos localizados en el proceso de excavación, no existiendo un muestreo específico dirigido al estudio de microvertebrados.

En este apartado solamente comentamos los resultados obtenidos en los depósitos de Santimamiñe, Kiputz IX, El Mirón, Cobrante y Antoliñako Koba. Este hecho se debe a diversos factores. En el caso de la cueva de Las Caldas, por el momento sólo se han publicado datos preliminares. En la monografía del yacimiento de Amalda, no se indica el número mínimo de individuos y por último, en Aitzbitarte III, en el proceso de cribado se empleó una luz de la malla de $1 \mathrm{~mm}$ (incluso en ocasiones puedo ser mayor), por lo que es posible que las especies más pequeñas estén infrarepresentadas.

En la única muestra procedente del nivel paleontológico de Santimamiñe (24956-24170 cal BP) dominan los espacios abiertos, destacando las especies de pradera (44\%) y de pradera húmeda (37\%).

Los resultados preliminares del nivel F de Kiputz IX reflejan un predominio de los espacios abiertos, aunque hay variaciones dentro de la dinámica de las especies de pradera. Con anterioridad a 24237-23330 cal BP (19970 \pm 150 BP $)$ la base de este nivel refleja un claro predominio de las especies que habitan en praderas húmedas (67\%), seguidas de las asociadas a zonas de ribera (24\%), pradera y bosque y/o matorral (4,5\%). Inmediatamente después a esta fecha, las especies de pradera aumentan en detrimento de las de praderas húmedas, dato que unido al ligero descenso de las especies de ribera, podría relacionarse con una regresión del grado de humedad. A partir de aqui y hasta un momento previo al 22084-21369 cal BP (18140 \pm 90 BP) tiene lugar una recuperación de las especies de pradera húmeda. Des- 
taca en la muestra 36, la aparición de Chionomys nivalis, especie asociada a contextos de montaña.

Al igual que sucedia en Kiputz IX, en la cueva de El Mirón dominan las especies de praderas. En el nivel solutrense más antiguo del estudio de micromamíferos (nivel 126: $23542-21638$ cal BP), el $88 \%$ corresponde a especies de praderas, dividiéndose por igual entre especies de pradera y de pradera húmeda. En el siguiente nivel (nivel 125: 23644-21709 cal BP) solamente existen especies de espacios abiertos, aunque ya ha comenzado la regresión de las de pradera. Es en este momento cuando las especies relacionadas con masas de agua no están presentes en el registro. El nivel 124, corresponde al periodo de mayor diversidad de hábitat y mayor número de individuos. Se mantiene el descenso, más atenuado, de especies de pradera y se da la única aparición de quirópteros. El grado de humedad aumenta en el nivel 123 , donde las especies de masas de agua y de praderas húmedas alcanzan un $87,5 \%$ (37,5 \% y $50 \%$, respectivamente). Esta tendencia se invierte en el nivel 122, donde los taxones de pradera aumentan un $28 \%$. La dinámica varía en el nivel 121 (22653-21213 cal BP) porque desaparecen los individuos de pradera y los de montaña aparecen con el valor porcentual más elevado de toda la secuencia (25\%), mientras que no hay grandes variaciones en el resto.

El yacimiento cántabro de Cobrante presenta tres niveles de cronología solutrense en los que predominan los micromamíferos relacionados con los espacios abiertos. Es el caso del nivel 4 (22146-21473 cal BP, $18260 \pm 70$ BP), en el que las especies de hábitats abiertos constituyen el $100 \%$ de la muestra, entre las cuales dominan las de pradera húmeda con un $70 \%$. En el nivel de contacto 3-4 unicamente se ha constatado la presencia de dos individuos, los cuales pueden ser relacionados con tres tipos de hábitat: masas de agua, pradera húmeda y pradera. Por último, el nivel 3 (22431-21602 cal BP, $18540 \pm 70$ BP), es el más interesante desde el punto de vista paleoambiental debido a la diversidad taxonómica (seis) y de tipos de hábitats, así como por el incremento del número de individuos. Los nueve individuos identificados presentan una clara preferencia hacia los espacios abiertos, con el $70 \%$ entre pradera húmeda y pradera. Un $20 \%$ están ligados a masas de agua, mientras que es en este nivel donde se ha identificado la única especie representativa de bosque y matorral. En esta ocasión, existe una diferencia entre los contextos ecológicos observados en los estudios de microfauna y palinológico. En el primero, se refleja un predomino de especies asociadas a paisajes abiertos, mientras que en el segundo, los valores porcentuales de la cubierta arbórea son los más elevados todos los registros comentados en este texto.

Las dos muestras del yacimiento de Antoliñako Koba, tienen un reducido número mínimo de individuos (muestra AK-6: 3 y muestra AK-5: 5). La muestra AK-6, adscrita al subnivel C2 (23435-22515 cal BP) conserva dos individuos de pradera húmeda y un individuo de bosque y matorral. En la muestra AK-5 (subnivel C1), también hay más individuos de pradera húmeda (3) que de bosque y matorral y pradera ( 1 individuo en cada grupo).

En todas las secuencias comentadas, incluso en la de la cueva de Amalda, predominan las especies de espacios abiertos. Entre las especies de pradera húmeda las más abundantes son Arvicola terrestris y Microtus (Alexandromys) oeconomus. Por su parte Microtus (Microtus) arvalis y Microtus (Microtus) agrestis son los que destacan entre el grupo de pradera.

Conviene recordar que algunos taxones como Erinaceus europaeus y Tálpidos suelen estar infra representados, debido a que son presas difíciles para las aves rapaces, generadoras de la mayoría de las acumulaciones de microvertebrados. Por ello, a pesar de que haya constancia de su presencia en gran número de registros (Balmori, Coberizas, La Riera, El Mirón, Morín, Aitzbitarte III, Aitzbitarte IV, Amalda, Ermittia, Antoliñako Koba y Kiputz IX), cabe la posibilidad que su representación paleoambiental no sea fiel a la realidad.

Del listado conjunto de taxones de estas secuencias solutrenses, únicamente Pliomys lenki y Microtus (Alexandromys) oeconomus no se encuentran en la actualidad en la región cantábrica. El primero está extinguido y el segundo, hoy en día, habita en zonas frías del centro y norte de Europa. Pliomys lenki, especie habitual a lo largo de todo el Pleistoceno superior en la cornisa cantábrica, solo se menciona en Las Caldas, El Mirón y Amalda.

En general, las condiciones ambientales serían frias, ya que junto a la presencia destacable de Microtus (Alexandromys) oeconomus resulta llamativa la escasa presencia de taxones indicadores de climas más cálidos como los géneros Apodemus, Eliomys y Glis.

En todos estos estudios, es evidente que el grupo más investigado ha sido el de los micromamíferos. En el Pais Vasco, los yacimientos de Antoliñako Koba y Santimamiñe son los únicos yacimientos donde existen referencias de restos de anfibios y reptiles: Anguis fragilis, cf. Podarcis y Rana temporaria-iberica en Antoliñako Koba y Rana temporaria en Santimamiñe. Por su parte, en Cobrante (Cantábria) los restos identificados en los niveles Solutrenses pertenecen a las especies Bufo calamita, Bufo sp. y Rana temporaria (Martín et al. 2009). Es aconsejable la incorporación de su estudio en futuras analíticas, dada la información paleoambiental y paleoclimática que también aportan. En un futuro próximo podremos disponer de los resultados sobre estos animales en el depósito de Kiputz IX.

\subsection{La dinámica paleoambiental durante el Solutrense}

A la hora de correlacionar los registros micropaleontológicos con las secuencias paleoambientales marinas y del 
Atlántico norte, el principal problema que se nos plantea es el de la cronología. Las complicaciones que se plantean al comparar dataciones radiocarbónicas efectuadas en medios tan dispares y con sus propias particularidades, dificultan la sincronización de los registros con la periodización paleoclimática. Teniendo en cuenta estas limitaciones, en esta exposición hemos optado por emplear fechas calibradas para poder relacionar, de modo tentativo, nuestros datos con los eventos paleoclimáticos del estadio isotópico 2 .

Los datos micropaleontológicos expuestos en el apartado 4 se concentran cronológicamente entre el 25000 y 20000 cal BP. La base del nivel F de Kiputz IX posiblemente se sitúe en el evento Heinrich 2, mientras que la fase más reciente del mismo corresponda ya a un periodo posterior. En su entorno medioambiental caracterizado por los espacios abiertos, con una escasa cubierta forestal (pino y a veces algo de enebro), los pequeños mamíferos reflejan variaciones en el grado de humedad, que por el momento no es posible definir con precisión en el paisaje vegetal, hasta que finalice el estudio palinológico. En cronologías similares, disponemos de los registros de La Riera, que se enmarcarían entre los eventos Heinrich 2 y 1 . Con posterioridad al evento Heinrich 2 hay constancia de momentos de mejoría climática, en los que aumenta el estrato arbóreo y los taxones caducifolios se recuperan y/o reaparecen. De todos modos, en este ámbito cronológico también se produjeron periodos de regresión climática, como sucede alrededor del 24672-22530 cal BP.

La base de la secuencia solutrense del yacimiento de El Mirón (23356-22546 cal BP) refleja unas condiciones frías y secas que se mantienen hasta la zona superior del nivel 126, cuando se invierte la tendencia y se detecta una mejora. En los niveles 125, 124 y 123 carecemos de información palinológica, pero las especies de micromamíferos reflejan el aumento del grado de humedad. Hacia el 22653-21213 cal BP destaca el aumento de los taxones de montaña en detrimento de las especies de pradera.

Durante la ocupación solutrense de la cueva de Amalda hay dos momentos paleoambientales. La zona inferior del nivel IV, mantiene la dinámica de regresión climática iniciada al final del nivel anterior. La mermada cubierta forestal, coincide con el bajo porcentaje de especies de micromamíferos asociados a entornos boscosos. Posteriormente, las condiciones mejoran, lo que supone un mayor desarrollo del bosque y de los elementos caducifolios y del grado de humedad.

A pesar de las limitaciones encontradas para poder definir la evolución paleoclimática durante este periodo del estadio isotópico 2 en la región cantábrica, sí podemos asegurar qué existieron variaciones medioambientales que influyeron en la dinámica de los diferentes taxones vegetales y faunísticos. Además, al sur de la Cordillera Cantábrica la humedad tuvo una influencia mucho mayor que en otras zonas del interior de la Península Ibérica (Gómez-Orellana 2002; Ramil-Rego et al. 2005).

\section{CONSIDERACIONES FINALES}

En la historia de las investigaciones prehistóricas en la región cantábrica destaca el interés de los arqueólogos por la evolución paleoambiental. A medida que se iban proponiendo diferentes aproximaciones analiticas, éstas iban siendo incorporadas a las actuaciones arqueológicas. No obstante, algunos de los criterios utilizados en su momento han supuesto que parte de los resultados obtenidos tengan una información sesgada. En general, el principal problema se localiza en la metodología empleada en el muestreo. Un intervalo de muestreo excesivamente amplio y/o una recogida parcial de los restos (empleo de luces de malla demasiado grandes para recuperar restos de tamaño muy pequeño) son factores determinantes de este proceso. No vamos a recordar de nuevo los condicionantes que nos hemos encontrado en algunos registros, pero dada la importancia de alguno de ellos, quizás resultaría aconsejable volver a muestrear la secuencia atendiendo los criterios metodológicos actuales.

A pesar de no disponer de secuencias continuas en los depósitos seleccionados, el predominio de los espacios abiertos queda reflejado tanto en el registro polínico como microfaunísticos, donde las especies de bosque están prácticamente ausentes. Incluso en los periodos más fríos, aunque los taxones vegetales asociados a la humedad puedan sufrir un retroceso, éste no fue lo suficientemente intenso como para permitir la expansión de los taxones estépicos. La ubicación geográfica de los sitios (ninguno de ellos alcanza los 400 metros de altitud y a excepción de El Mirón y Lezetxiki, todos los demás están relativamente próximos al mar) es un factor a tener en cuenta en la caracterización de este paisaje. No existen discordancias entre los resultados procedentes tanto de contextos arqueológicos como de los depósitos de origen no antrópico (Kiputz IX y Río Bóo).

\section{AGRADECIMIENTOS}

Este estudio ha sido subvencionado por los proyectos HAR 2010-22013 del MEC, Unesco 09/01, EHU08/84, y EHU10/32 de la Universidad del País Vasco/Euskal Herriko Unibertsitatea. También los Grupos de Investigación IT-28807/ UFI 11-09 del Gobierno Vasco y GUI12/35 de la UPV/ EHU. Uno de los autores (N. G-I) disfruta de una beca de formación de investigadores (BFI-2010-289/AE) del Gobierno Vasco. Agradecemos a A. Prieto de Dios su colaboración en este artículo. • 


\section{BIBLIOGRAFÍA}

AgUIRRE, M. 2000: "El yacimiento paleolítico de Antoliñako koba (Gautegiz-Arteaga, Bizkaia): secuencia estratigráfica y dinámica industrial. Avance de las campañas de excavación 1995-2000". Illunzar 4: 39-81.

AltunA, J. 1970: "Fauna de Mamiferos del Yacimiento Prehistórico de Aitzbitarte IV (Rentería-Guipúzcoa)". Munibe 22: 3-41.

- 1971: "Los mamíferos del yacimiento prehistórico de Morín (Asturias)". En J. González Echegaray y L. G. Freeman (eds.): Cueva Morín. Excavaciones 1966-1968. Publicaciones del Patronato de las Cuevas prehistóricas de la provincia de Santander. Santander: 369-383.

- 1972: Fauna de mamíferos de los yacimientos prehistóricos de Guipúzcoa. Con Catálogo de los Mamíferos Cuaternarios del Cantábrico y del Pirineo Occidental. Munibe 24.

- 1986: "The mammalian faunas from the prehistoric site of La Riera". En L. G. Strauss y G. A. Clark (eds.): La Riera Cave. Stone age hunter gatherer adaptations in Northern Spain. Anthropological Research Papers, 36. Arizona: 237-331.

Altuna, J., Baldeon, A. y Mariezkurrena, K. (eds.) 1990: La cueva de Amalda (Zestoa, País Vasco). Ocupaciones paleolíticas y postpaleolíticas. Colección Barandiarán 4. Eusko Ikaskuntza. Donostia.

AltunA, J., MarieZKurRenA, K. y Rios, J. (eds.) 2011: Ocupaciones humanas en Aitzbitarte III (País Vasco). 33.600-18.400 BP (zona de entrada a la cueva). Serie EKOB - Colección de Patrimonio Cultural Vasco 5. Gobierno Vasco. Vitoria-Gasteiz.

Arrizabalaga, A., Altuna, J., Areso, P., Falgueres, C., Iriarte, M.J., Mariezkurrena, K., Peman, E., Ruiz-Alonso, M., Tarriño, A., Uriz, A. y VALLVERDÚ, J. 2005: "Retorno a Lezetxiki (Arrasate, Pais Vasco): nuevas perspectivas de la investigación". En M. Santonja, A. Pérez-Gónzalez y M. J. Machado (eds.): Geoarqueología y Patrimonio en la Península lbérica y el entorno mediterráneo. ADEMA. Soria: 81-98.

Barandiarán, J. M. De 1925: "Pueblo de Kortezubi (Bizkaia). Barrios de Basondo y Terliz. Los establecimientos humanos y las condiciones naturales". Anuario de Eusko Floklore V: 45-67.

- 1947: "Prehistoria de Vizcaya, un cuarto de siglo de investigaciones". Ikuska 4-5: 134-147.

- 1961: "Excavaciones en Aitzbitarte IV. Trabajos de 1960". Munibe XIII (3-4): 183-245.

- 1963a: "Excavaciones en la caverna de Aitzbitarte IV. Trabajos de 1961". MunibeXV (1-2): 23-42.

- 1963b: "Excavaciones en Aitzbitarte IV. Campaña de 1962". Munibe XV (3-4): 69-86.

- 1964: "Excavaciones en la caverna de Aitzbitarte IV. Campaña de 1963". Munibe XVI (1-2): 12-23.

- 1965: "Excavaciones en Aitzbitarte IV. Campaña de 1964". Munibe XVII (1-4): 21-37.

- 1976: Vasconia antigua. Tras las huellas del Hombre (III). La cueva de Santimamiñe. Obras Completas tomo IX. Biblioteca de la Gran Enciclopedia Vasca. Bilbao.

BarAndiarán, J. M. y Altuna, J. 1970: "Excavación de la cueva de Lezetxiki (Campaña de 1968). Munibe 22: 51-59.

BoYER-KLeIN, A. 1980: "Nouveaux résultats cantabriques au Tardiglaciaire". Bulletin de la Societé Préhistorique française 77/4: 103-107.

- 1984: "Analyses polliniques cantabriques au Tardiglaciaire". Revue de Paleobiologie vol.spec: 33-39.

BRoNk, C. 2009: "Bayesian analysis of radiocarbon dates». Radiocarbon $51,337-360$

BuRJachs, F., López-Sáez, J. A. e IrIARTE, M. J. 2003: "Metodología arqueopalinológica". En R. Buxó y R.Piqué (dir.): La recogida de muestras en arqueobotánica: objetivos y propuestas metodológica. Museu d'Arqueologia de Catalunya. Girona: 11-18.

CABrerA, V. 1977: "El yacimiento solutrense de Cueva Chufin (Ciclones, Santander)". Actas del XIV Congreso Nacional de Arqueología (Vitoria 1975): 157-164.
Castaños, P. 2005: "Cueva de Kiputz IX (Mutriku). I Campaña". Arkeoikuska 2004. Servicio Central de Publicaciones del Gobierno Vasco. Vitoria-Gasteiz: 141-143.

- 2006: "Cueva de Kiputz IX (Mutriku). II Campaña". Arkeoikuska 2005. Servicio Central de Publicaciones del Gobierno Vasco. VitoriaGasteiz: 136-138.

- 2007: "Cueva de Kiputz IX (Mutriku). III Campaña". Arkeoikuska 2006. Servicio Central de Publicaciones del Gobierno Vasco. VitoriaGasteiz: 190-192.

- 2008: "Cueva de Kiputz IX (Mutriku). IV Campaña". Arkeoikuska 2007. Servicio Central de Publicaciones del Gobierno Vasco. VitoriaGasteiz: 376-378.

ClaRK, G. A. 1974: "Excavations in the Late Pleistocene cave site of Balmori. Asturias (Spain)". Quaternaria 18: 383-426.

Corchón, M. S. 1981: Cueva de Las Caldas. Excavaciones Arqueológicas en España 115. Madrid.

- 1995: "La cueva de Las Caldas (Priorio, Oviedo). III: resultados preliminares de las excavaciones (campañas 1991-1994)". Excavaciones arqueológicas en Asturias: 1991-94: 45-60.

Cuenca Bescós, G., Straus, L.G., González Morales, M. y Garcia Pimienta, J. C. 2008: "Paleoclima y paisaje del final del cuaternario en Cantabria: los pequeños mamíferos del Mirón". Revista Española de Paleontología 23: 91-126.

- 2009: "The reconstruction of past environments through small mammals: from the Mousterian to the Bronze Age in El Mirón Cave". Journal of Archaeological Science 36: 947-955.

DUPRÉ, M. 1988: Palinología y Paleoambiente. Nuevos datos españoles. Referencias. Serie Trabajos Varios 84. Servicio de Investigación Prehistórica. Diputación Provincial de Valencia.

- 1990: "Análisis polínico de la cueva de Amalda". En J.Altun., A.Baldeon y K.Mariezkurrena (eds.): La Cueva de Amalda (Zestoa, País Vasco). Colección Barandiarán 4. Eusko Ikaskuntza. Donostia: 49-51.

Gómez-Orellana, L. 2002: El último ciclo Glaciar-Interglaciar en el litoral del NW ibérico. Dinámica climática y paisajistica. Tesis Doctoral. Universidad de Santiago de Compostela. Lugo.

González EchegaraY, J. 1980: El yacimiento de la cueva de El Pendo Excavaciones (1953-1957). Biblioteca Praehistórica Hispana 17. Consejo Superior de Investigaciones Científicas. Madrid.

González Echegaray, J., Garcia Guinea, M. A. y Begines, A. 1966: Cueva de El Otero. Excavaciones Arqueológicas en España 53. Madrid.

González Echegaray, J. y Freeman, L. G. (eds.) 1971: Cueva Morin: excavaciones 1966-1968. Publicaciones del Patronato de las cuevas prehistóricas de la provincia de Santander.

IRIARTE-CHIAPUSSO, M. J. 2011a: "El medio vegetal del yacimiento de Aitzbitarte III (Renteria, Pais Vasco), a partir de su análisis palinológico". Serie EKOB - Colección de Patrimonio Cultural Vasco 5. Gobierno Vasco. Vitoria-Gasteiz: 57-78.

- 2011b: "Polen y vegetación en la secuencia estratigráfica de Santimamiñe (Kortezubi, Bizkaia)". En López Quintana, J. C. (dir): La cueva de Santimamiñe: revisión y actualización (2004-2006). Kobie Serie Excavaciones Arqueológicas en Bizkaia 1, Diputación Foral de Bizkaia. Bilbao: 321-342.

- 2012: "The vegetational contexts of El Mirón cave (Ramales de la Victoria, Cantabria) throughout the early-mid Holocene: contributions from the palynological study". En L. G. Straus y M. R. González Morales (eds.): El Mirón Cave: Cantabrian Spain. The Site and its Holocene Archaeological Record. University of New Mexico Press. Albuquerque: 119-142.

laplana, C., Sevilla García, P., lópez Martínez, N. y Corchón Rodriguez, M. S. 2006: "Primeros datos sobre los micromamíferos (Roedores, Insectivoros, Quirópteros y Lagomorfos) del Solutrense (Pleistoceno superior final) de la Cueva de Las Caldas (Oviedo, Asturias)". En E. Fernández-Martínez (ed.): XXII Jornadas de la Sociedad Española de Paleontología y simposios de los proyectos PICG 493, 503, 499, y 467. Universidad de León. León: 137-139. 
Lerol-Gourhan, Arl. 1971: "Análisis polínico de Cueva Morin". En J. Gonzalez Echegaray y L. G. Freeman (eds.): Cueva Morin: excavaciones 1966-1968: 359-365. Patronato de las Cuevas Prehistóricas. Santander: $359-365$.

- 1980: "Analyse pollinique de la grotte du Pendo". En J. González Echegaray: El yacimiento de la cueva de El Pendo. Biblioteca Praehistorica Hispana XVII: 265-266.

- 1986: "The palynology of La Riera Cave". En L. G. Straus y G. A. Clark (eds.): La Riera Cave. Anthropological Research Papers 36.Tempe: 59-64.

López-Garcia, J. M. 2008: Evolución de la diversidad taxonómica de los micromamíferos en la Península lbérica y cambios paleoambientales durante el Pleistoceno superior. Tesis Doctoral. Universitat Rovira i Virgili.

López Quintana, J. C. (2011): La cueva de Santimamiñe: revisión y actualización (2004-2006). Kobie Serie Excavaciones Arqueologica en Bizkaia 1. Diputación Foral de Bizkaia, Bilbao.

Maillo, J. M., Valladas, H., Cabrera, V. y Bernaldo De Quirós, F. 2001: "Nuevas dataciones para el Paleolítico superior de Cueva Morin (Villanueva de Villaescusa, Cantabria)". Espacio, Tiempo y Forma. Serie I. Prehistoria y Arqueología 14: 145-150.

Martin, C., SZYndalaR, Z. y SANCHIZ, B. 2009: "Herpetofauna del Pleistoceno Superior de la cueva de Cobrante". En P. Rasines del Rio (dir.): Arqueología en la Cueva de Cobrante (Cantabria, España). Sautuola 15: 107-114.

Murelaga, X., Ballón, S., Sáez De lafuente, X., Castaños, P., López Quintana, J.C., Guenaga lizasu, A., Ortega, L., Zuluaga, M. C. y Alonso Olazabal A. 2011: "La Fauna de microvertebrados de Santimamiñe (Pleistoceno superior-Holoceno) (Kortezubi, Bizkaia)" En J. C. López Quintana (dir.): La cueva de Santimamiñe: revisión y actualización (20042006). Kobie Serie Excavaciones Arqueologica en Bizkaia 1. Diputación Foral de Bizkaia. Bilbao: 291-320.

Paquereau, M. 1981: "Análisis palinológico". En S. Corchón. Cueva de Las Caldas, Excavaciones Arqueológicas en España 115. Madrid: 50-51.

Peman, E. 1990: "Los micromamíferos de la cueva de Amalda y su significado. Comentarios sobre Pliomys lenki (Heller, 1930) (Rodentia, Mammalia)". En J. Altuna, A. Baldeón y K. Mariezkurrena (eds.): La Cueva de Amalda (Zestoa, País Vasco). Ocupaciones Paleolíticas y Postpaleolíticas. Fundación Jose Miguel de Barandiarán. Eusko Ikaskuntza. Donostia: 225-238.

- 2011: "Los micromamíferos del yacimiento de Aitzbitarte III (Rentería, Gipuzkoa) (Zona de Entrada). Serie EKOB - Colección de Patrimonio Cultural Vasco 5. Gobierno Vasco. Vitoria-Gasteiz: 57-78.
Ramil-Rego, P., Iriarte, M. J., Muñoz-Sobrino, C. y Gómez-Orellana, L. 2005: "Cambio climático y dinámica temporal del paisaje y de los hábitats en las ecorregiones del NW de la Península lbérica durante el Pleistoceno superior". Munibe Homenaje a J. Altuna 57 (1): 537-551.

Reimer, P. J., Baillie, M. G., Bard, E., Bayliss, A., Beck, J. W., Blackwell, P. G., Bronk Ramsey, C., Buck, C. E., BurR, G., Edwards, R. L., Friedrich, M., Grootes, P., Guilderson, T., Hajdas, I., Heaton, T. J., Hogg, A. G., Hughen, K. A., Kaiser, K. F., Kromer, B., Mccormac, E. G., Manning, S. W., Reimer, S. D., Richards, D. A., Southon, J., Talamo, S., Turney, C., Van Der Plicht, J. y WEYHENMEYER, C. E. 2009: "Intcal09 and Marine09 radiocarbon age calibration curves, 0-50 000 years cal BP". Radiocarbon 51 (3): 1111-1150.

RuIz ZaPATA, B. y GIL-Garcia, M. J. 2009: "El paisaje vegetal de los alrededores de la cueva de Cobrante: análisis palinológico". En P. Rasínes del Rio (dir.): Arqueología en la Cueva de Cobrante (Cantabria, España). Sautuola 15: 55-62.

SÁnCHEZ-GoÑı, M. F. 1993: De la taphonomie pollinique à la reconstitution de l'environment. L'exemple de la region cantabrique. BAR International Series 586, Oxford.

SESÉ, C. 2009: "Los micromamiferos (Rodentia, Insectivora y Lagomorpha) del yacimiento del Pleistoceno Superior de la cueva de Cobrante". En P. Rasínes del Rio (dir.): Arqueología en la Cueva de Cobrante (Cantabria, España). Sautuola 15: 85-100.

Straus, L. G. y Clark, G. A. (EDS.) 1986: La Riera Cave. Stone age hunter gatherer adaptations in Northern Spain. Anthrop. Papers 36. Arizona State University.

Straus, L.G., Gónzalez, M. R., GutiérRez, I. e Iriarte Chiapusso, M. J. 2011 : "Further Solutrean evidence in El Mirón cave (Ramales de la Victoria, Cantabria)". Munibe 62: 117-133.

Straus, L.G., González Morales, M. R., Marin Arroyo, A. B. e Iriarte-ChiAPUSSO, M. J. EN PRENSA: "The human occupations of El Mirón cave (Ramales, Cantabria, Spain) during the last glacial maximum/solutrean period". Congreso Internacional El Solutrense (Almeria). Espacio Tiempo y Forma. Serie I, Prehistoria y Arqueología 4.

ZUBELDIA, H. 2006: Goi Pleistozenoko-Behe Holocenoko Antoloñako koba aztarnategiaren mikroornodunen ikerketa. Tesina. Euskal Herriko Unibertsitatea EHU/UPV.

Zubeldia, H., Murelaga, X., Bailón, S., Aguirre, M. y Sáez De lafuente, X. 2003-2007: "Micorvertebrados de la secuencia superior de Antoliñako Koba (Pleistoceno superior) (Gauteguiz-Arteaga, Bizkaia)". Kobie XXVII: 5-49. 